Instituto Internacional de Investigación y Desarrollo Tecnológico Educativo INDTEC, C.A.

DOI: https://doi.org/10.29394/scientific.issn.2542-2987.2017.2.3.3.53-75

OAI-PMH: http://www.indteca.com/ojs/index.php/Revista Scientific/oai

\title{
Experiencia Previa al Modelo de Prácticas de la UNAE
}

Autores: Ormary Egleé Barberi Ruiz Universidad Nacional de Educación, UNAE ormary.barberi@unae.edu.ec Cuenca, Ecuador María Dolores Pesántez Palacios Universidad Nacional de Educación, UNAE maria.pesantez@unae.edu.ec

Chuquipata, Ecuador

\section{Resumen}

Los planteamientos contenidos en el presente artículo representan una versión preliminar que fundamentará el modelo de prácticas pre profesionales (PPP) de la Universidad Nacional de Educación (UNAE) del Ecuador, imperiosa necesidad institucional, revelada en los análisis descriptivos realizados a partir de los soportes técnicos-administrativos (informes, entrevistas, testimonios), fundamentos pedagógicos de la UNAE (direccionalidad curricular, ejes transversales de la práctica, plan de carrera, Aproximación y Exploración diagnóstica como asignatura de naturaleza para la praxis pre profesional) y la demanda de los contextos socio educativos donde se han realizado las prácticas que emerge para redimensionarla. Relacionar estos elementos permitió proyectar aspectos de la modelación de los procesos de la práctica pre profesional para el desarrollo de las competencias profesionales del futuro docente, a través de cuatro componentes: proyectivo contextual, implementación (tutorización), acompañamiento (pareja pedagógica) y seguimiento (encuentros al inicio, durante y final de la práctica). La formación inicial del docente es inherente a la docencia (formación académica y profesional), la investigación y la vinculación con la colectividad, pilares éstos fundamentales de la educación superior ecuatoriana.

Palabras clave: prácticas pre profesionales; modelo pedagógico; direccionalidad curricular. 


\title{
Previous Experience a Model of Practice UNAE
}

\begin{abstract}
The statements presented in this article represents a preliminary version of the proposed model of pre-professional practices (PPP) of the National University of Education (UNAE) of Ecuador, an urgent institutional necessity is revealed in the descriptive analyzes conducted from technical support administrative (reports, interviews, testimonials), pedagogical foundations of UNAE (curricular directionality, transverse axes in practice, career plan, approach and diagnostic examination as subject nature of the pre professional practice) and the demand of socio educational contexts where the practices have been emerging to resize them. By relating these elements allowed conceiving the modeling of the processes of the pre-professional practices for the development of professional skills of future teachers through four components: contextual projective, implementation (tutoring), accompaniment (teaching couple) and monitoring (meetings at the beginning, during and end of practice). The initial training of teachers is inherent to teaching (academic and professional training), research and links with the community, these are fundamental pillars of Ecuadorian higher education.
\end{abstract}

Keywords: pre-professional practices; pedagogical model; curriculum directionality.

Date Received: $18-08-2016$

Date Acceptance: 22-09-2016 


\section{Introducción}

Es pertinente abordar la temática de las prácticas pre profesionales en la formación inicial del docente, desde la panorámica de los sistemas educativos de las naciones de América Latina, pese a sus iniciativas y esfuerzos siguen en deuda con sus sociedades en cuanto al compromiso de brindar una educación de calidad para todos y todas, cuestionada en diversos escenarios a través de informes de encuentros de embajadores educativos de la región, temas debatidos en eventos educativos y de interés en numerosas investigaciones al respecto.

Entre los aspectos que han sido objeto de análisis en los escenarios referidos, predomina la relación entre la calidad educativa con el desempeño profesional del docente, revelando contradicciones significativas del proceso de formación inicial de los docentes por parte de las Instituciones de Educación Superior (IES). Sin embargo, no es la intención soslayar los avances específicos alcanzados en la materia, como la recopilación titulada "Estudio de Casos de Modelos Innovadores en la Formación Docente en América Latina y Europa" (OREALC/UNESCO, 2006) el cual contiene un conjunto de experiencias innovadoras consolidadas en materia de formación docente.

En relación al escenario planteado, la generalidad de las IES públicas en Ecuador, incluyendo las de formación docente, persisten en modelos pedagógicos tradicionales caracterizados por una docencia conductista y tecnocrática, anclada en el estereotipo del profesor universitario que cree tener el poder y control del conocimiento ante el estudiante inexperimentado, según lo concluido por (Cartuche, N, y otros, 2015); obviando la pedagogía basada en metodologías creativas, innovadoras y transformadoras para facilitar nuevos conocimientos y experiencias de aprendizajes a partir de la unidad teoría y práctica desde, en y para la práctica docente.

Dadas las circunstancias educativas descritas, se promulga el Proyecto de Ley de creación de la Universidad Nacional de Educación (UNAE), 
(Registro Oficial, 2013), desde fundamentos contemplados en la Constitución de la República del Ecuador, como en las leyes orgánicas de la Educación Intercultural, la normativa en materia de Educación Superior y el Plan Nacional del Buen Vivir (Comisión Gestora, 2015), como un componente estratégico sobre la base de principios constitucionales, filosóficos y pedagógicos para impulsar las condiciones que deriven en una mejora de la calidad educativa a partir de la formación inicial del docente.

En otras palabras, la UNAE representa un eje estratégico para dinamizar los procesos de transformación del sistema educativo ecuatoriano a través de una formación docente de alto impacto por medio de procesos de aprendizajes de calidad que conlleven a la transformación sustancial del pensamiento, la formación y la práctica docente. Esta argumentación se relaciona con lo planteado en la introducción del artículo "Paradojas y Contradicciones en la Formación de Maestros a partir del Buen Vivir" (Álvarez, $F, 2016)$.

En este sentido, la Comisión Gestora de la UNAE, asume la notable tarea de elaborar el modelo pedagógico; aprobado junto a los proyectos de las carreras: Educación Básica (EB), Intercultural Bilingüe (EIB) y Educación Inicial (EI); con sus respectivas especificaciones a saber: niveles de formación de la educación superior, la organización de los aprendizajes a partir de los componentes: docencia, prácticas de aplicación y experimentación y aprendizaje autónomo (CES, 2013). Estos elementos en la dinámica de formación deben guardar estrecha relación en favor a una formación inicial docente innovadora y transformadora, con énfasis en la práctica pre profesional.

A la luz del análisis de los elementos referidos a la organización de los aprendizajes, es conveniente abordar teórica y contextualmente la práctica pre profesional desde la normativa educativa ecuatoriana y desde el modelo pedagógico de la UNAE. La normativa establecida en el Reglamento de 
Régimen Académico (RRA), modificado del año 2014, define la práctica pre profesional como "....actividades de aprendizaje orientadas a la aplicación de conocimientos y al desarrollo de destrezas y habilidades específicas que un estudiante debe adquirir para un adecuado desempeño en su futura profesión" (CES, 2013).

El precepto normativo citado, confiere una definición general y esencial sobre las prácticas pre profesionales en cuanto a elementos del perfil requerido para el desempeño docente que ha de considerarse en la formación profesional "aplicación de conocimientos, habilidades y destrezas"; sin embargo, no considera especificaciones de aspectos sobre el sentir, pensar y relaciones socio afectivas del estudiante en formación como producto de las vivencias y experiencias significativas que derivan de la práctica sobre contextos educativas reales y que privilegian la construcción de sus capacidades y vocación como futuro profesional de la docencia.

En cuanto a los elementos esenciales para modelar la formación del estudiante que cursa una de las carreras de estudio en la UNAE, a saber son: las competencias, incluyendo las relaciones socio afectivas y sentir del que aprende; la práctica como escenario para lograr concretar las teorías y metodologías útiles en la estudio de situaciones, casos y problemas educativos reales, sobre la base de experiencias sentidas y reflexionadas; la cooperación permanente en el proceso de formación; el aprendizaje y servicio para facilitar el compromiso ético y social del aprendiz; la evaluación formativa como proceso que facilita la metacognición del estudiante en formación (Comisión Gestora, 2015).

La práctica pre profesional en el contexto UNAE, es considerada desde el inicio de las diferentes carreras que imparte, orientada hacia la reflexión y confrontación permanente entre la teoría y las prácticas escolares cotidianas para el desarrollo de las competencias profesionales; así también, constituye el eje integrador de las experiencias de aprendizaje del futuro docente 
(Comisión Gestora, 2015). También, su enfoque integrador responde a la vinculación con la demanda de las asignaturas y el componente investigativo, en función a los sujetos, casos, situaciones y problemas educativos reales que existen en las instituciones educativas.

Entre los principios orientadores de la práctica se tienen los siguientes: aprender haciendo, priorizar los contenidos (esencializar el currículo); considerar las metodologías aprendizajes como: lesson study, aula invertida, estudios de casos, problemas o proyectos; integrar los contextos reales y virtuales para responder a la demanda de la educación en la era digital, destinando el contexto presencia de aprendizaje a resolver dudas, inquietudes, problemas, diseño y ejecución de proyectos; fomentar un clima de confianza y cooperación donde todos aprenden; y potenciar la interculturalidad como fuente de diálogo abierto y constructivo.

Así también, el modelo pedagógico de la UNAE, deriva en una estructura curricular concebida principalmente desde, en y para la práctica, destinando el $40 \%$ del currículo de formación al desarrollo de actividades prácticas en conjunto con el componente teórico y virtual provoca una dinámica integradora de conocimientos para privilegiar la reconstrucción del pensamiento práctico de los futuros docentes a partir de las experiencias significativas, evidenciándose la relevancia que tiene para la formación inicial del docente las experiencias de aprendizaje en situaciones educativas reales, requiriendo la integración de la teoría y la práctica. (Comisión Gestora, 2015)

La teoría como componente curricular del referido modelo permite comprender, cuestionar y diseñar la práctica, sobre la base de espacios de plataformas virtuales, para conformar el $60 \%$ restante del currículo. La teoría un espacio de estudio, reflexión, debate e indagación sobre la práctica antes, durante y después de la participación del estudiante en procesos de observación, diagnóstico, análisis, reflexiones, diálogos, valoraciones y 
creaciones sobre situaciones, casos y problemas auténticos, presentes en las instituciones educativas donde se realizan las prácticas pre profesionales.

Para atender a otras especificaciones descriptivas del modelo pedagógico que tributan a su direccionalidad curricular, se debe considerar los componentes de la formación docente a partir de las interrogantes claves contenidas en los núcleos problémicos y los ejes transversales de cada ciclo de estudio; éstos elementos direccionan el conjunto sistémico de las asignaturas que a su vez responden a diversos campos de formación: teóricametodológica, praxis pre profesional, epistemología y metodología de la investigación, comunicación y lenguaje, idioma. (UNAE, 2015)

Las especificaciones curriculares referidas deben propiciar en la dinámica formativa de las prácticas pre profesionales la adquisición de experiencias significativas sobre la base de los ejes medulares del prácticum: desarrollo del pensamiento crítico, teorización de la práctica, experimentación de la teoría y comprensión de la acción (Pérez, A, 2012). Procesos estos que demandan una tutorización por parte del docente de prácticas para provocar niveles graduales y sistemáticos de análisis y reflexión (crítica y autocrítica) en el estudiante en formación.

Desde el análisis realizado al Modelo Pedagógico de la UNAE, las autoras del presente artículo, consideran que la ejecución curricular a partir de los campos de formación, de sus elementos transversales, de los principios pedagógicos y de la práctica pre profesional, se funde en una dinámica sistémica de formación, proporcionándole al aprendiz durante y/o al final de la carrera:

- Competencias básicas y profesionales sobre la base de un modelo pedagógico con una estructura curricular equilibrada en escenarios de aprendizaje teórico, práctico y virtual para facilitar el desarrollo de contenidos conceptuales, procedimentales y actitudinales (con énfasis 
en sus emociones y sentimientos); derivando en la práctica maneras de pensar, hacer y actuar del estudiante, a partir de procesos graduales e inherentes a explicar, investigar y resolver problemas educativos en los diferentes niveles del sistema educativo ecuatoriano;

- Niveles de comprensión para caracterizar la práctica docente en una realidad educativa compleja, cambiante, incierta y exigente que demanda de una atención pedagógica creativa, innovadora y transformadora acorde a los diversos contextos socio-culturales;

- Escenarios para responder a la demanda tecnológica, utilizando herramientas y metodologías pedagógicas pertinentes a los tiempos de la era digital y al modelo de sociedad;

- Procesos de formación práctica de niveles de complejización gradual en el transcurrir de la carrera, los mismos permitirán profundizar desde el análisis, la reflexión crítica y autocrítica sobre situaciones, casos y problemas educativos, a través de procesos complejos y permanentes que le consoliden como docentes investigadores de su propia práctica.

Las autoras al relacionar los fundamentos pedagógicos antes referidos y los aspectos normativos contenidos en el RRA sobre el componente de la praxis pre profesional, con la dinámica real de formación inicial del docente en la UNAE, desarrollada en el periodo académico de abril a julio del año 2016, en las carreras de EB, EIB y El, les permite afirmar que existen limitaciones relevantes en cuanto a la correspondencia entre los componentes: docencia, investigación y la práctica pre profesional, debido a la débil direccionalidad curricular de los procesos de formación entre los componentes mencionados, específicamente en la práctica pre profesional.

Exponiendo el escenario en cuestión con otras palabras, la direccionalidad curricular debe constituirse en un proceso consciente de los 
docentes que imparten las diferentes asignaturas, especialmente las de naturaleza práctica y que guardan estrecha relación con los ejes transversales, es decir que al diseñar y ejecutar las actividades de aprendizajes de cada componente, el docente debe planificar, ejecutar y evaluar el proceso de aprendizaje según los elementos curriculares establecidos para cada ciclo en la malla curricular respectiva.

Si bien, el plan de práctica pre profesional elaborado para el periodo académico mencionado, representó un punto de partida significativo para la vinculación entre los contextos socio-educativos y la UNAE, además de constituir un documento de orientación general a disposición del docente de práctica; se debe valorar críticamente su alcance curricular y su carácter general $o$ de estandarización, sin considerar las particularidades y correspondencia entre los elementos medulares de cada carrera que se imparte en relación a la dinámica que demanda las diferentes instituciones educativas (necesidades, intereses, situaciones educativas, socio-culturales, problemas, entre otros).

Por lo anteriormente señalado, se puede afirmar que las prácticas pre profesionales en la UNAE se han desarrollado con aciertos y desaciertos, significativos en ambas vertientes, propio de un proceso en plena iniciación y construcción para alcanzar la idoneidad y pertinencia de sus elementos medulares en la formación inicial del docente en un novel contexto universitario, en este caso con especial atención a los aspectos y criterios que se deben considerar para el diseño, implementación, acompañamiento y seguimiento de las prácticas pre profesionales en el contexto UNAE.

Como precedente se puede hacer referencia a los primeros momentos de las prácticas pre profesionales como elemento vinculador entre las demandas curriculares (asignaturas) y la realidad escolar, es decir las prácticas estaban al servicio de las asignaturas. Posteriormente, esta relación fue revertida, producto de una valoración institucional y lineamientos oficiales, 
las prácticas permiten recoger las demandas de las instituciones educativas para luego teorizarlas dentro de la universidad (López, M, 2015).

A partir de las aseveraciones señaladas, no se pretende desconocer los esfuerzos humanos y materiales destinados hasta el momento para el perfeccionamiento del componente de la praxis pre profesional en la formación inicial docente que imparte la UNAE, por el contrario constituyen las contradicciones y desvinculaciones entre los elementos curriculares y procesos esenciales de la práctica, evidenciadas en el periodo académico recién finalizado, un punto de partida para los análisis realizados desde una reflexión profunda sobre la base de los fundamentos normativos, contextuales y pedagógicos que enmarcan dicha formación docente. Algunos resultados del análisis serán expuestos en la metodología del presente artículo.

Los planteamientos expuestos, permitieron introducir argumentos de la situación contradictoria evidenciada en el proceso de la práctica pre profesional en la UNAE en cuanto a la débil direccionalidad según los elementos curriculares de la malla (núcleos problémicos y ejes transversales) en cada ciclo de la carrera; además de lo coherencia y lógica de los procesos requeridos para la observación, diagnóstico y experimentación de la práctica (niveles de gradualidad). Estos escenarios inciden en la calidad de la formación inicial del docente.

En consecuencia a lo expuesto, son formuladas las siguientes interrogantes: ¿Cómo fundamentar la práctica pre profesional de manera consecuente con los elementos, principios y ejes transversales del modelo pedagógico de la UNAE para incidir en la formación del futuro docente investigador que requiere el sistema educativo ecuatoriano?, ¿De qué manera proyectar, desarrollar, acompañar y evaluar la práctica pre profesional como eje integrador de la formación inicial del docente?.

Para dar respuesta a estas formulaciones surge la necesidad de iniciar la creación del modelo de prácticas pre profesionales de manera coherente, 
sistemática y pertinente a las dinámicas requeridas para la modelación de los procesos que facilitan el desarrollo de las competencias específicas del estudiante en formación. Avances iniciales al respecto, contenidos en el presente artículo para proyectar la modelación en el proceso de formación desde la práctica.

\section{Metodología}

Como sustento a las aseveraciones planteadas es ético y pertinente señalar las fuentes y evidencias que permitieron describir los aspectos más significativos de la práctica pre profesional durante el periodo académico abril - julio 2016. A continuación, se especifican:

- Visitas a las instituciones educativas al final de la práctica, lo cual permitió un diálogo con las autoridades educativas institucionales, y recabar sus impresiones sobre el desarrollo de la misma.

- Análisis de los reportes presentados en los informes consignados por los docentes responsables de la práctica en el periodo académico anterior, en cuanto a las actividades realizadas, objetivos alcanzados, conclusiones y recomendaciones.

- Encuentros con las autoridades educativas, especialmente algunos directores de carrera y docentes de la asignatura de aproximación o exploración diagnóstica y cátedra integradora; para socializar y unificar criterios del proceso de inicio de la práctica en el periodo académico septiembre 2016 a febrero 2017.

Con los datos recabados se realizó un análisis descriptivo de los aspectos favorables y desfavorables más significativos que caracterizaron la práctica pre profesional durante el periodo académico referido. Para tal fin se contó con la participación del equipo de la dirección de prácticas pre 
profesionales de la UNAE, implicados en el proceso de cierre de la práctica del ciclo académico abril-julio 2016, lo que permitió una visión desde adentro por parte de los autoras, facilitándose la comunicación directa con los actores involucrados en el proceso de práctica, tanto del contexto UNAE como las instituciones educativas; así también disponer de los soporte técnicos administrativos que avalan el desarrollo y resultados de la misma.

A partir de las fuentes de información sobre la práctica pre profesional de la UNAE, anteriormente señaladas, se presentan aspectos del análisis que la describen a continuación:

- Las prácticas pre profesionales se llevaron a cabo bajo la modalidad regular, intensiva y de inmersión en diversas instituciones educativas (24), ubicadas en su mayoría en Azogues y otras en el Cañar, durante el periodo abril-julio 2016, con la participación de los practicantes distribuidos en un total de 19 paralelos del 1ero al 3er ciclo, adscritos a las diversas carreras como: EI, EIB, EE Y EB.

- En cuanto al proceso de inducción al inicio de las prácticas, fue dirigida por la coordinación respectiva de la UNAE a docentes responsables y autoridades de las instituciones educativas, a quienes se socializó información general y el plan de prácticas unificado como referencia para las carreras. Al respecto, hubo algunos reportes que indicaron desinformación por parte del docente de aula en cuanto a aspectos de la práctica, incluyendo su participación; debido a la omisión por parte de las autoridades educativas y del profesor de práctica en la difusión de la inducción, lo que generó descoordinación con el trabajo de los estudiantes.

- Afirmaciones generales sobre la relevancia y éxito de las PPP, a través de lo manifestado por los estudiantes relacionado a lo aprendido de los 
docentes en ejercicio: aumento de la motivación profesional, la comprensión y diagnóstico de situaciones educativas, un aprender haciendo, los aprendizajes significativos que generó el ejercicio de acercamiento a la lesson study. Sin embargo, no se reportaron situaciones específicas de aprendizaje en función a la práctica, según el nudo problémico y ejes transversales del ciclo respectivo, como tampoco especificaciones de los estudiantes sobre los niveles de reflexión crítica alcanzado, experiencias adquiridas en la teorización de la práctica y experimentación de la teoría.

- Interesantes experiencias en las prácticas pre profesional de inmersión con resultados relevantes en el aprendizaje pre profesional en cuanto a lo pedagógico e investigativo en la comunidad (UEIB Sisid); sin embargo, testimonios recogidos por parte de los líderes comunitarios afirman que faltó una adecuada vinculación de las practicantes en la dinámica comunitaria.

- Discontinuidad en el proceso de las prácticas pre profesional en las instituciones educativas (observar, ayudar, acompañar y experimentar), por actividades establecidas o no en el cronograma para evaluaciones parciales y la tendencia de suspender clases los días viernes para realizar actividades sociales de diferente índole.

- Escaso intercambio durante el desarrollo de las prácticas pre profesionales entre el docente de práctica y los docentes de aula. Así también fue escaso, el intercambio con el docente de cátedra integradora para los ajustes sobre la dinámica del proceso.

- Pese a la disponibilidad de un plan general de prácticas pre profesionales, estandarizado para las diversas carreras, se evidenció en los reportes de los informes consignados pocas coincidencias con las actividades establecidas por semana en el plan respectivo, dando 
como resultado la falta de coherencia entre el plan y la ejecución de la misma.

- En la revisión de los informes finales a la Dirección de prácticas pre profesionales se evidenciaron algunas elaboraciones que no siguieron los criterios orientados para su elaboración, inclusive unos pocos no identificaron datos claves y otros pocos presentaron una breve descripción general de las prácticas pre profesionales.

El análisis de los reportes más significativos obtenidos en el informe final señalado y el intercambio de la dirección de prácticas de la UNAE con algunas autoridades, directores de carrera y docentes de las asignaturas Aproximación o Exploración Diagnóstica y Cátedra Integradora, permitió recabar una serie de recomendaciones durante el periodo académico que recién inicia:

- Los docentes responsables de la práctica pre profesional deben ser docentes del paralelo respectivo, es inconveniente que se asignen tutores que desconozcan a los estudiantes.

- Responder a la demanda e interés de formación continua que expresan algunos docentes de las instituciones educativas.

- Es importante garantizar un proceso de inducción apropiado a cada ciclo y al nivel de formación de los practicantes, ya que en algunos escenarios de la práctica pre profesional los docentes de aula aspiran que éstos asuman mayor responsabilidad pedagógica durante la rutina escolar.

- Organizar las prácticas pre profesionales en correspondencia con los meses más estables en cuanto a la dinámica y rutina escolar de las instituciones educativas. Inclusive en algunos casos se expresa la 
tendencia de que los días viernes son suspendidas con frecuencia las actividades escolares.

- Insistir en la importancia de una buena comunicación fluida y permanente entre el docente de prácticas y los docentes en ejercicio, crear un clima de confianza para alcanzar unas prácticas de mayor coordinación y en ambiente de cooperación. Inclusive se evidenció que la inducción dirigida a los rectores, en algunos casos no fue replicada a los docentes de aula, quienes a veces ignoraban especificaciones de su participación en el proceso.

- En la inducción que se realiza a los estudiantes de la UNAE antes de iniciar las prácticas pre profesionales, se debe promover efectivamente el compromiso y empoderamiento de su formación como docente, para minimizar algunas actitudes que distan de una buena disposición y accionar como practicante.

- Se reportó la necesidad de ajustar los instrumentos de observación y diagnóstico a elementos, procesos, contenidos, relacionados a los ejes transversales y nudo problémico de cada ciclo.

- Se requiere que los docentes responsables de la práctica pre profesional, precisen los criterios e indicadores de evaluación en correspondencia a la direccionalidad de cada ciclo (por ejemplo, criterios e indicadores para la elaboración del portafolio, la reflexión sobre la práctica e informe final).

- Escasos reportes sobre los resultados del espacio destinado para la reflexión de los practicantes sobre la práctica vivenciada en las instituciones educativas, menos aún se precisaron reportes de los avances sobre el desarrollo del pensamiento crítico de los estudiantes y la comprensión de la acción docente. 
- Precisar orientaciones de cómo deben participar los docentes de aula y las autoridades educativas en el desarrollo de la práctica, específicamente el proceso de evaluación de las mismas, en virtud de que se evidenció en los reportes que pocas veces son considerados y otras veces no.

- Consignar ante la dirección de la institución educativa una copia del informe final de práctica pre profesional, elaborado por los practicantes que contenga las fortalezas, debilidades y sugiera propuestas y recomendaciones a dicho centro.

- Brindar especificaciones sobre los resultados de la evaluación de las prácticas pre profesionales, ya que no se evidencia en los informes detalles al respecto.

Es necesario que en las prácticas pre profesionales del periodo académico septiembre 2016 a febrero 2017, superen no solo las debilidades expresadas anteriormente y potencien los aspectos favorables, atendiendo a las sugerencias y recomendaciones referidas, sino también se consideren los resultados del análisis a partir de los alcances curriculares desde la práctica, soslayados hasta ese momento.

De esta manera, se atendería desde la dirección de prácticas pre profesionales de la UNAE, la descontextualización y desarticulación a partir de los elementos curriculares contenidos en el modelo pedagógico y en los micro curriculums de cada carrera, para establecerse una organización sistemática de las experiencias de aprendizaje de manera secuencial, gradual e integradora que procure la adquisición no solo de contenidos conceptuales, sino también procedimentales y actitudinales (valores, sentimientos, emociones) y en consecuencia durante la carrera el aprendiz alcanzará las 
competencias profesionales fundamentales para desempeñarse en la labor docente con calidad y excelencia.

Para tal fin, se considera una prioridad la creación del modelo de prácticas pre profesionales que si bien, no es el momento para presentar sus especificidades se pueden proyectar de manera general sus componentes esenciales para elevar su coherencia, pertinencia y coordinación práctica, sobre la base de la direccionalidad pedagógica curricular que se omite en la experiencia desarrollada en los ciclos anteriores.

\section{Resultados}

La aproximación a un modelo de la práctica pre profesional en la UNAE, en su versión preliminar debe representar un sistema de componentes que permitan instrumentar su dinámica, para que los estudiantes al final de la carrera alcancen las competencias profesionales que le permitan atender las necesidades y problemas educativos, según las particularidades de cada carrera; sobre la base de las deficiencias obtenidas a partir del análisis realizado, de tal manera que se pueda facilitar un proceso de modelación de la práctica pre profesional más coherente en función a:

- Concebir los fundamentos teóricos y contextuales en correspondencia a la demanda del sistema educativo ecuatoriano, al Plan Nacional del Buen Vivir y al modelo pedagógico UNAE.

- Proyectar la práctica a partir de un diseño que considere la correspondencia de los elementos transversales de las mallas curriculares de cada carrera, la naturaleza de las asignaturas que en su dinámica de formación propicien en menor o mayor grado la integración de saberes para el desarrollo del pensamiento crítico, teorizar la práctica, experimentar la teoría y comprender la acción. Así también, la proyección del carácter investigativo de la práctica. 
- Redimensionar la tutorización por parte del docente responsable de la práctica para que el estudiante en formación vivencie y adquiera experiencias significativas, acompañe, ayude, experimente e investigue situaciones, casos y problemas educativos. Estos procesos se irán complejizando conforme avance la carrera.

- Coordinar la implementación de la práctica desde los aspectos técnico - administrativo, pedagógico (syllabus de Aproximación y/o Exploración diagnóstica en correspondencia a la asignatura Cátedra Integradora, criterios para el trabajo de la pareja pedagógica) y la demanda en los contextos educativos en donde se realiza la práctica para validar los saberes, para facilitar el desarrollo de competencias específicas en cada carrera, construcción de métodos y técnicas de investigación (observación, diagnóstico, estudio de casos, propuesta, proyectos, entre otros).

- Redimensionar el proceso de implementación de la práctica por medio del monitoreo, acompañamiento y seguimiento valorativo a partir de lo proyectado en cuanto a los aspectos técnico - práctico.

Los aspectos señalados corresponden a los componentes del modelo de las prácticas pre profesionales desde una concreción teórica y práctica para concebir los procesos de modelación desde una fundamentación pedagógica, curricular, investigativa y de vinculación con la colectividad que en el plano práctico se concreta en una dinámica sistémica de experiencias de aprendizaje sobre la base de la reflexión y confrontación teórico y práctica, para que el estudiante en formación desarrolle gradualmente las competencias requeridas para su futuro desempeño como docente en los contextos educativos ecuatorianos. 


\section{Conclusiones}

El modelo pedagógico de la UNAE enmarca la formación del futuro docente ecuatoriano a partir de los fundamentos, principios y elementos transversales que derivan en una estructura curricular que privilegia a las prácticas pre profesionales como el componente curricular de mayor relevancia en el proceso de formación docente por competencias, como resultado de la dinámica integradora que propicia la aplicación de conocimientos, habilidades, actitudes, emociones y valores por parte de los estudiantes, desde el inicio de la carrera hasta su culminación.

Las prácticas constituye el eje integrador de las experiencias de aprendizaje, sobre la base de vivencias en contextos educativos reales que demandan del estudiante procesos de búsqueda continua por medio de la reflexión de su propia práctica, experimentación de la teoría y la comprensión de la acción, desde escenarios socio-educativos que requieren de procesos progresivos inherentes a la investigación acción y a la vinculación con la comunidad para validar los saberes y teorías, derivando en mejoras progresivas en su propio proceso de aprendizaje y enseñanza en las diversas asignaturas.

Dada la trascendencia de las prácticas pre profesionales en la calidad de la formación docente y ante el reto que representa para la UNAE como universidad emblemática del Ecuador, son significativas las reflexiones y análisis sobre el desarrollo de las prácticas contenidas en el presente artículo, permitiendo concretar acciones desde la Dirección de Prácticas, para garantizar niveles de direccionalidad del proceso de formación profesional e investigativo, hacia las competencias profesionales a través de los ejes transversales y los nudos problémicos, elementos establecidos en los micro currículos de cada carrera de estudio.

Así también, los resultados de las reflexiones y análisis presentados sobre las prácticas, revelan la necesidad de modelar desde un enfoque 
sistémico los procesos de formación desde, en y para la práctica pre profesional a partir de su correspondencia con el modelo pedagógico y curricular de la UNAE para el desarrollo de competencias de manera gradual, sistemática y coordinada, requeridas para un desempeño docente y profesional de calidad y de excelencia en el nivel educativo ecuatoriano que corresponda.

Las relaciones de correspondencia entre los elementos esenciales del modelo pedagógico y curricular de la UNAE con el proceso de prácticas pre profesionales, permitió proyectar aspectos del modelo de prácticas a favor de procesos de formación práctica (aplicabilidad y experimentación ) sobre la base de experiencias de aprendizaje que contribuyen en el desarrollo de las competencias profesionales del futuro docente, a través de cuatro componentes esenciales: proyectivo contextual (programa de prácticas por carrera, sílabo, plan de clase de la pareja pedagógica), implementación de la práctica (tutorización, tensiones educativas), acompañamiento (pareja pedagógica en cada ciclo, conformada por los docentes de las asignaturas Cátedra Integradora y Aproximación o Exploración diagnóstica ) y seguimiento (redimensionamiento de la práctica a partir de encuentros al inicio, durante y final de la práctica.

\section{Referencias}

Álvarez, F. (2016). Paradojas y contradicciones en la formación de maestros a partir del Buen Vivir. Ecuador: UNAE.

Cartuche, N. y otros (2015). El modelo pedagógico en la práctica docente de las universidades públicas del país. Ecuador: s/e.

CES (2013). Reglamento de Régimen Académico. Quito: s/e.

Comisión Gestora (2015). Modelo pedagógico UNAE. Azogues: s/e.

López, M. (2015). Prácticas Pre Profesionales. En UNAE, Hacer bien, pensar, bien y sentir bien. Azogues: UNAE. 
OREALC/UNESCO (2006). Modelos Innovadores en la Formación inicial docente. Chile.

Pérez, A. (2012). La formación inicial del profesorado basado en competencias. La relevancia del prácticum. Taller 4. Ecuador. [Documento en línea]. Recuperado de:

http://www.ces.gob.ec/doc/8tavoTaller/el\%20practicum.pdf

Registro Oficial (2013). Ley de Creación de la Universidad Nacional de Educación. UNAE. Quito: s/e.

UNAE (2015). Malla curricular de carrera. Azogues: s/e.

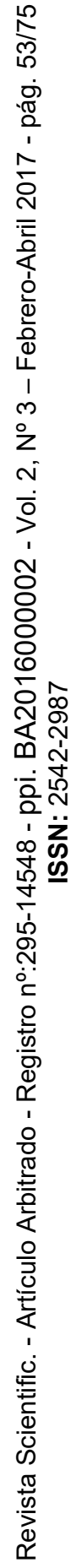

73 


\section{Ormary Egleé Barberi Ruiz \\ e-mail: ormary.barberi@unae.edu.ec}

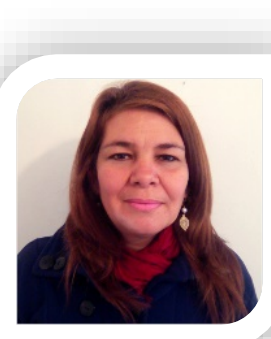

Doctora en Ciencias Pedagógicas. Especialización en Desarrollo Comunitario. Licenciada en Educación Integral, Mención Ciencias Naturales y Salud. Experiencia profesional en docencia, dirección y supervisión de instituciones educativas e instancias administrativas del sistema educativo venezolano. Docente jubilada del Ministerio de Educación-Venezuela. Docente por horas de pregrado y postgrado en la Universidad Pedagógica Experimental Libertador-UPEL y Universidad Nacional de los Llanos Occidentales Ezequiel Zamora-UNELLEZ. Ha participado como ponente en eventos nacionales e internacionales. Actualmente, se desempeña como docente-investigadora en la Universidad Nacional de Educación (UNAE) en Ecuador. Es miembro activo de la Línea de Investigación: Desarrollo profesional de los docentes. 


\section{María Dolores Pesántez Palacios}

e-mail: maria.pesantez@unae.edu.ec

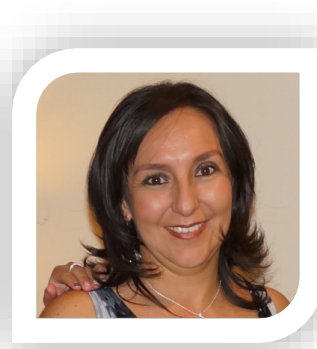

Licenciada en Tecnologías de Estimulación Temprana en Salud, Ciencias de la Educación con mención en Pedagogía, Máster en Educación Parvularia, títulos alcanzados en la Universidad de Cuenca, Universidad Politécnica Salesiana y Universidad ARCIS de Chile. Docente en la Universidad de Cuenca en las facultades de Ciencias Médicas en la carrera de Estimulación Temprana y Psicología en la carrera de Psicología Educativa. En la Universidad Politécnica Salesiana, docente en la carrera de parvularia. Docente Investigadora, directora de Vinculación con la colectividad, prácticas pre profesionales y la revista de divulgación y experiencias pedagógicas MAMAKUNA, en la Universidad Nacional de Educación UNAE.

El contenido de este manuscrito se difunde bajo una Licencia de Creative Commons ReconocimientoNoComercial-Compartirlgual 4.0 Internacional 